\title{
The recurrent ultra-luminous X-ray transient NGC 253 ULX1
}

\author{
M. Bauer and W. Pietsch \\ Max-Planck-Institut für extraterrestrische Physik, Giessenbachstraße, 85741 Garching, \\ Germany \\ email: mbauer@mpe.mpg.de
}

\begin{abstract}
We present the results of ROSAT and XMM-Newton observations of the recurrent ultraluminous X-ray source (ULX) NGC 253 ULX1. This transient is one of the few ULXs that was detected during several outbursts. The luminosity reached $1.4 \times 10^{39} \mathrm{erg} \mathrm{s}^{-1}$ and $0.5 \times$ $10^{39} \mathrm{erg} \mathrm{s}^{-1}$ in the detections by ROSAT and XMM-Newton, respectively, indicating a black hole $\mathrm{X}$-ray binary (BHXRB) with a mass of the compact object of $>11 \mathrm{M}_{\odot}$. In the $R O S A T$ detection NGC 253 ULX1 showed significant variability, whereas the luminosity was constant in the detection from XMM-Newton. The XMM-Newton EPIC spectra are well-fit by a bremsstrahlung model $\left(\mathrm{kT}=2.24 \mathrm{keV}, N_{H}=1.74 \times 10^{20} \mathrm{~cm}^{-2}\right)$, which can be used to describe a comptonized plasma. No counterpart was detected in the optical I, R, B, NUV and FUV bands to limits of 22.9, 24.2, 24.3, 22 and $23 \mathrm{mag}$, respectively, pointing at a XRB with a low mass companion.
\end{abstract}

Keywords. X-rays: binaries - X-rays: individuals: (NGC 253 ULX1) - black hole physics accretion, accretion disks.

\section{Introduction}

ULXs are extra-nuclear compact X-ray sources with luminosities considerably exceeding the Eddington luminosity for stellar mass X-ray binaries of $\sim 2 \times 10^{38} \mathrm{erg} / \mathrm{s}$ (Makishima et al. 2000). ULXs obtain special attention as they may indicate the existence of intermediate mass black holes $\left(100-1000 \mathrm{M}_{\odot}\right.$, IMBHs). The ULX presented here, NGC 253 ULX1, was found by Liu \& Bregman (2005) searching for ULXs in ROSAT HRI observations of 313 nearby galaxies. We analysed the source for variability (Fig. 1) and extracted the first spectrum from XMM-Newton data. For a more extensive report on NGC 253 ULX1 see Bauer \& Pietsch (2005).

\section{Properties of NGC 253 ULX1}

NGC 253 ULX1 showed two outburst. The first was observed by ROSAT with $L_{X}=$ $1.4 \times 10^{39} \mathrm{erg} / \mathrm{s}(0.3-10 \mathrm{keV})$, the second was detected by XMM-Newton with $L_{X}=$ $0.5 \times 10^{39} \mathrm{erg} / \mathrm{s}(0.3-10 \mathrm{keV})$. From the first outburst we determined a lower mass limit of the compact object to $11 \mathrm{M}_{\odot}$. We were able to obtain the first spectrum of this source from the second outburst. It was best fitted with a bremsstrahlung model $(\mathrm{kT}=2.24 \mathrm{keV}$, $\left.N_{H}=1.74 \times 10^{20} \mathrm{~cm}^{-2}, \chi_{\text {red }}^{2}=0.961\right)$. The brightness of the source varies by at least a factor of $500\left(L_{X, \min }=0.003 \times 10^{39} \mathrm{erg} / \mathrm{s}\right)$. Its fastest change in luminosity $\left(L_{\max } / L_{\min }\right)$ is greater than 71 in 120 days. In the ROSAT observation, NGC 253 ULX1 showed significant variability by at least a factor of 2 during 8 days. No short term variability was detected in the XMM-Newton observation.

\section{Optical counterpart}

We checked images taken with the Wide Field Imager (WFI) on the MPG-ESO $2.2 \mathrm{~m}$ telescope at La Silla in the R-, I- and B-band (limiting magnitudes 24.2, 22.9 and 24.3, 

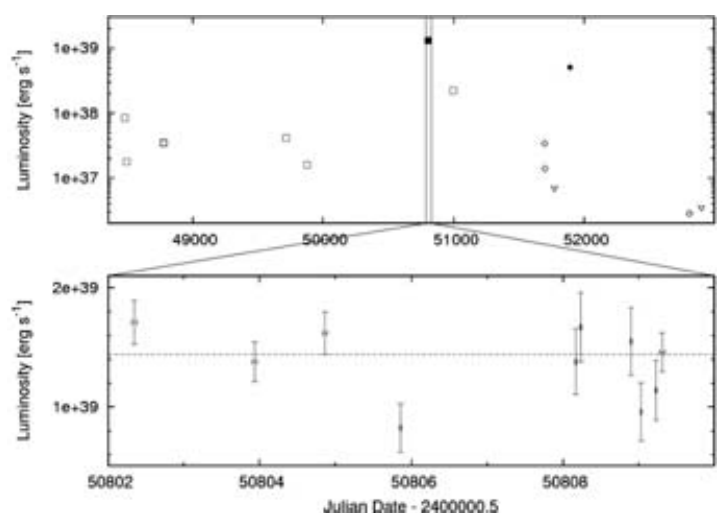

Figure 1. NGC 253 ULX1 light curves. Upper panel: solid symbols represent detections, open symbols $3 \sigma$ upper limits of NGC 253 ULX1. Different symbols represent observing instruments: ROSAT (squares), XMM-Newton (diamonds) and Chandra (triangles). Lower panel: Luminosities of individual ROSAT HRI exposures from observation $601111 \mathrm{~h}$. In contrast to the upper panel, in the lower panel the plot is linear in luminosity.

respectively) and images taken with the Galaxy Evolution Explorer (GALEX, a space telescope from NASA observing in the ultraviolet) in the NUV and FUV (limiting magnitudes 22 and 23, respectively), but no counterpart could be detected.

\section{The nature of NGC 253 ULX1}

The best fitting bremsstrahlung spectrum indicates that NGC 253 ULX1 is a XRB system. We determined the lower mass limit of the compact object from its ROSAT luminosity to $11 \mathrm{M}_{\odot}$, typical for a stellar mass black hole. If we assume a multicolour disk blackbody model (XSPEC model diskbb, fitted with $N_{H}=1.30 \times 10^{20} \mathrm{~cm}^{-2}$, $\left.\mathrm{kT}=0.62 \mathrm{keV}, \chi_{\text {red }}^{2}=1.671\right)$ then NGC 253 ULX1's position in the luminosity-disk temperature diagram (Fig. 2 in Miller et al. 2004) also indicates that NGC 253 ULX1 is a stellar mass black hole. The lack of an optical counterpart points at a low mass companion in the system. A high mass XRB should have been detectable in the WFI images (see Sect. 3) at about 22 to 24 mag (extrapolating V magnitudes from high mass XRBs in the Magellanic Clouds, Liu et al. 2000).

The recurrent outbursts exclude that the source is the luminous remnant of a recent supernova, like e.g. SN1993J in M81 (Zimmermann \& Aschenbach 2003). We can also exclude that NGC 253 ULX1 is either a foreground object or a background active galactic nucleus (AGN) based on three arguments: (i) Its $\log \left(f_{\mathrm{x}} / f_{\text {opt }}\right)$ value of $>3.2$ exceeds that expected for galactic sources $(-4.6$ to -0.6$)$ as well as AGNs $(-1.2$ to +1.2$)$ (Maccacaro et al. 1988). (ii) The variability of NGC 253 ULX1 is by far larger than typically observed for AGNs ( 10-60) and (iii) NGC 253 ULX1 shows a bremsstrahlung spectrum, whereas spectra of AGNs above $2 \mathrm{keV}$ are typically fitted by a power law.

We conclude that NGC 253 ULX1 is a low mass XRB with a stellar black hole as compact companion.

\section{References}

Bauer, M. \& Pietsch, W. 2005, astro-ph/0507248, A\&A accepted

Liu, J. \& Bregman, J. N. 2005, ApJS, 157, 59

Liu, Q. Z., van Paradijs, J., \& van den Heuvel, E. P. J. 2000, A\&AS, 147, 25

Maccacaro, T., Gioia, I. M., Wolter, A., Zamorani, G. \& Stocke, J. T. 1988, ApJ, 326, 680

Makishima, K., Kubota, A., Mizuno, T., et al. 2000, ApJ, 535, 632

Miller, J. M., Fabian, A. C. \& Miller, M. C. 2004, ApJ, 614, L117

Zimmermann, H.-U. \& Aschenbach, B. 2003, A\&A, 406, 969 